\title{
BMJ Open Risk and protective factors of dementia among adults with post-traumatic stress disorder: a systematic review protocol
}

\author{
Karen A Lawrence (D) , ${ }^{1}$ Theresia M Pachner, ${ }^{1}$ Molly M Long (D) , ${ }^{1}$ \\ Stephanie Henderson, ${ }^{2}$ Donna L Schuman, ${ }^{1}$ Brenda L Plassman ${ }^{3}$
}

To cite: Lawrence KA, Pachner TM, Long MM, et al. Risk and protective factors of dementia among adults with post-traumatic stress disorder: a systematic review protocol. BMJ Open 2020;10:e035517. doi:10.1136/ bmjopen-2019-035517

- Prepublication history and additional material for this paper are available online. To view these files, please visit the journal online (http://dx.doi. org/10.1136/bmjopen-2019035517).

Received 11 November 2019 Revised 03 March 2020 Accepted 01 May 2020

Check for updates

(c) Author(s) (or their employer(s)) 2020. Re-use permitted under CC BY-NC. No commercial re-use. See rights and permissions. Published by BMJ.

${ }^{1}$ College of Social Work, University of Kentucky, Lexington, Kentucky, USA ${ }^{2}$ Medical Center Library, University of Kentucky, Lexington, Kentucky, USA

${ }^{3}$ Department of Neurology, Duke University School of Medicine, Durham, North Carolina, USA

Correspondence to

Dr Karen A Lawrence;

karen.lawrence@uky.edu

\section{ABSTRACT}

Introduction Post-traumatic stress disorder (PTSD) is associated with an increased risk of dementia. Individual epidemiological studies have controlled for several confounders of the relationship between PTSD and increased dementia risk, yet particular risk factors underlying this relationship have not been determined. This systematic review protocol aims to identify risk and protective factors of dementia among adults with PTSD. Methods and analysis We will conduct an electronic search of the databases: PubMed, CINAHL, PsychINF0, The Cochrane Library, Scopus and ProQuest Dissertation and Theses Global. After screening the studies, quantitative synthesis will be performed, if possible. Otherwise, a narrative synthesis will be performed. We will include randomised controlled trials and other types of research evidence including longitudinal cohort studies. Strength of evidence will be assessed using the Grading of Recommendations, Assessment, Development and Evaluations method. Examples of variables that will be extracted are: year of PTSD diagnosis, comorbid conditions, health behaviours, pharmacological treatments and year of mild cognitive impairment or dementia diagnosis. We developed this systematic review protocol according to the Preferred Reporting Items for Systematic Review and Meta-Analysis Protocols 2015 statement. Ethics and dissemination The proposed study will not collect individual-level data and, therefore, does not require ethical approval. Results of this study will provide current evidence on risk and protective factors of dementia in adults with PTSD. Findings will be disseminated in peerreviewed publications and conference presentations. PROSPERO registration number CRD42019128553.

\section{INTRODUCTION}

Post-traumatic stress disorder (PTSD) is a psychiatric syndrome that may develop in individuals who have experienced a traumatic event ${ }^{1}$ (eg, war, sexual assault, accident or environmental catastrophe) resulting in symptoms of intrusive thoughts, avoidance of trauma-related stimuli, negative alterations in cognition or mood and changes in arousal. ${ }^{2}$ PTSD is characterised clinically by four groups of related symptoms, also known as 'symptom clusters', including: (1) intrusive thoughts

\section{Strengths and limitations of this study}

- This systematic review will provide a comprehensive search of the literature regarding risk and protective factors of dementia among adults with post-traumatic stress disorder (PTSD).

- Experts in the field of cognitive decline, mild cognitive impairment, all-type dementia and PTSD developed and guided the search strategy.

- An exhaustive search of multiple databases (ie, PubMed, CINAHL, PsychINF0, The Cochrane Library, Scopus and ProQuest Dissertation and Theses Global) will be performed to ensure a comprehensive review, yet, potentially relevant articles from other/additional databases may be missed.

- Given that this review will be restricted to observational studies with 300 or more participants and randomised controlled trials with 50 or more participants, relevant studies with important evidence may be missed, for example, from studies with smaller samples.

- Another potential limitation may be that a metaanalysis may not be conducted due to included studies being insufficiently homogenous, in which case a narrative approach will be used.

(eg, feelings of re-experiencing the event, memories, dreams or flashbacks), (2) avoidance of trauma-related stimuli (eg, distressing internal reminders such as thoughts, memories or feelings and distressing external reminders such as people, places, conversations or objects), (3) negative alterations in cognition or mood (eg, inability to remember aspects of the traumatic event, negative beliefs about self or others, persistent negative emotional state, feelings of detachment or anhedonia) and (4) changes in arousal (eg, irritable or angry outbursts, exaggerated startle response, hypervigilance, problems concentrating, sleep disturbance). ${ }^{2}$ Overall, about 8.6 million US adults or $5.7 \%$ of the population, aged $18-64$ years, are diagnosed with PTSD at some point in their lifetime. ${ }^{34}$ Research over the last decade has indicated 
that a subset of individuals with PTSD is at greater risk for developing all-type dementia.

Epidemiological evidence from studies of military veterans ${ }^{5-7}$ and civilians ${ }^{89}$ has revealed that individuals with PTSD have up to a fourfold greater risk of developing dementia than those without PTSD. However, it remains unclear how PTSD leads to cognitive decline and development of dementia. Within the general population, experts have identified several protective factors associated with decreased dementia risk (eg, physical activity, cognitive training, Mediterranean diet and vegetable intake). ${ }^{10}$ Such health behaviours may be more common in adults with PTSD who do not develop mild cognitive impairment (MCI) or dementia. Conversely, factors associated with increased dementia risk, such as genetic factors (eg, being homozygous for APOE $\varepsilon 4$ ), negative health behaviours (eg, tobacco use), psychiatric disorders (eg, major depressive disorder), use of certain pharmaceutical medications (eg, benzodiazepines) and medical history (eg, stroke), ${ }^{11}$ may occur more frequently in persons with PTSD who develop MCI or dementia.

However, it is also possible that PTSD confers neurobiological changes that contribute to the pathogenesis of dementia. While an association between PTSD and impaired cognitive performance has been established ${ }^{12-16}$ whether the cognitive effects of PTSD symptoms are mechanistically linked to the later development of dementia is a significant gap in knowledge. In prior retrospective cohort research on dementia incidence in veterans with and without PTSD that controlled for several confounding factors, findings indicated that a history of head injury, substance misuse and clinical depression were not responsible for the increased dementia risk in persons with PTSD. ${ }^{6}$ Interestingly, a prospective cohort study of World Trade Center first-responders who developed PTSD revealed that the intrusive thoughts, symptom cluster of PTSD, was consistently predictive of cognitive impairment 14 years later. ${ }^{17}$ This finding suggests that exposure to PTSD itself may be a risk factor for dementia. It remains unclear, however, which particular risk factors account for the increased risk of dementia in the PTSD population. Addressing this knowledge gap, we will examine exposure to PTSD symptoms and previously identified risk and protective factors associated with MCI and all types of dementia among those with PTSD.

In a conceptualisation of cognition as a continuum of indiscrete, overlapping degrees of functional ability, normal cognition would sit at the left end, followed by cognitive decline, MCI, and non-specific dementia and Alzheimer's disease (AD) at the right end of the continuum. Experts consider some degree of cognitive decline as a normal part of the ageing process. MCI is set apart from normal age-related cognitive decline in that the degree of decline is greater than expected, considering the person's age and educational background, yet impairment is not severe enough to meet criteria for a diagnosis of dementia. Extant research on the stages of cognitive decline leading to dementia indicates that as early as mid-life, alterations in the brain are detectable by imaging and other biomarkers ${ }^{18-20}$ introducing a potential opportunity for targeting modifiable risk and protective factors of dementia. However, the implementation of screening and detection of cognitive decline in the PTSD population as a means to facilitate early detection and delay or prevention of dementia is another area in need of research.

Researchers in the field of geriatric mental health have identified several gaps in the care of older adults with PTSD. Relative to younger adults, PTSD among older adults is under-identified, under-treated and underresearched. ${ }^{21}$ Furthermore, conventional evidence-based PTSD treatments do not specifically focus on maintaining cognitive health. Given the increased prevalence of dementia in the PTSD population in conjunction with the fact that brain changes associated with cognitive decline start long before symptoms are evident, ${ }^{22}$ an assessment of risk and protective factors could be used by clinicians to augment PTSD treatment of middle-aged adults in service of supporting cognitive health. Thus, the overarching research question to be addressed is: 'What are risk and protective factors of dementia among adults with PTSD?'

\section{Objectives}

This systematic review aims to critically appraise the research on risk and protective factors of $\mathrm{MCI} /$ dementia among adults with PTSD. Potential and anticipated risk factors may include well-known comorbidities of PTSD, such as major depressive disorder, ${ }^{23}$ substance use disorder, ${ }^{24}$ obesity ${ }^{25}$ and other associated genetic or biological factors (ie, APOE genotype ${ }^{26}$ or female sex, ${ }^{27}$ respectively). Potential and anticipated protective factors may include cognitive training or physical exercise. Several of the aforementioned factors, among others, are also associated with risk and prevention of dementia, respectively. ${ }^{28-36}$ Therefore, the following two questions will be addressed within a comparative context. Among adults with PTSD, comparisons will be made between those who did and did not develop MCI or dementia.

1. What are the recency, severity and duration of PTSD exposure?

2. What is the exposure (dose, frequency, duration) of previously identified risk and protective factors of dementia?

\section{METHODS AND ANALYSIS}

\section{Protocol and registration}

This systematic review will be based on the approach proposed by Joanna Briggs Institute ${ }^{37}$ and will build on a prior systematic review of risk and protective factors for dementia among the general population. ${ }^{10}{ }^{37}$ The former describes an approach toward systematic reviews, in which a search is conducted for the 'best available' evidence.

The search strategy will be guided by the Preferred Reporting Items for Systematic Review and Meta-Analysis 
Protocols (PRISMA-P) ${ }^{38}$. PRISMA-P is a search protocol for systematic reviews and meta-analyses, which includes an evidence-based minimum number of items for reporting. The search strategy (see online supplementary appendix A) aims to locate both published and unpublished studies, as well as grey literature. Medical subject headings and text words related to PTSD, cognitive decline, MCI, dementia, and risk and protective factors (based on a prior systematic review by Plassman and colleagues ${ }^{10}$ ) were used to develop a search strategy.

\section{Search for and identification of studies}

The following databases will be searched: PubMed, CINAHL, PsychINFO, The Cochrane Library and Scopus. Additionally, we will search ProQuest Dissertations and Theses Global for grey literature and unpublished studies. We will limit our literature search to the English language and human subjects. To guarantee literature satisfaction, the references of included articles, identified through the search, will be scanned.

\section{Eligibility criteria}

Studies on adults, with an average age of 50 years and older at follow-up, who have been diagnosed with PTSD will be included, given that neurodegenerative changes can be observed before the age of $60 .{ }^{3940}$ Because the identified problem is the increased prevalence of dementia in older adults with PTSD, comparators are adults aged 50 years or over with PTSD (current or past) who do not develop MCI or dementia. All racial and ethnic populations will be considered.

Epidemiological findings have shown that chronic stress and PTSD are both associated with an increased incidence of different types of dementia. ${ }^{167}$ Therefore, this study will assess risk and protective factors for MCI and all frequent/common causes of dementia of all possible aetiologies, including senile dementias, vascular dementia, $\mathrm{AD}$, frontotemporal dementia, Lewy body dementia and dementia not otherwise specified. This review will consider observational longitudinal cohort studies with 300 or more participants and randomised controlled trials (RCTs) with 50 or more participants, in order to include studies with sufficient statistical power. ${ }^{37}$ In addition, for observational studies, documentation of cognitive changes over at least 1 year will be required for studies on MCI, to provide time for the occurrence of clinically meaningful change. ${ }^{1037}$ Two years will be required for studies of all types of dementia, including $\mathrm{AD}^{1037}$ because of the lengthy prodromal phase associated with dementia, especially AD. The study outcomes will be a diagnosis of MCI or any type of dementia, using acceptable standards. Given that PTSD was accepted as a disorder and added to the Diagnostic and Statistical Manual of Mental Disorders by the American Psychiatric Association in $1980,{ }^{41}$ this systematic review will consider any studies published from 1980 until 29 February 2020.

\section{Patient and public involvement}

There was no patient or public involvement in the development of this study protocol.

\section{Study selection and data abstraction}

Using Covidence software ${ }^{42}$ and following the protocol of Williams and colleagues ${ }^{37}$ two reviewers with a third tie-breaker reviewer will autonomously screen the titles and abstracts yielded by the searches against the inclusion and exclusion criteria. Articles meeting the inclusion criteria will subsequently undergo a full-text screening performed by three reviewers. The three reviewers will read each article and, based on this screening, render a decision regarding which articles meet the inclusion criteria. Disagreements will be resolved by consensus. The review will be completed by 15 December 2020 .

\section{Data collection process and data items}

Data extraction will be performed in duplicate and data will be input into evidence tables in Excel, modelled on the format of Williams and colleagues. ${ }^{37}$ The data elements to be extracted include descriptors to assess applicability, quality elements, intervention and exposure details, and outcomes. More specifically, the data items will include: details about the timing of diagnosis and duration of follow-up for PTSD, MCI and dementia diagnoses and other cognitive outcomes; assessment/ diagnostic instruments used for PTSD and all cognitive outcomes; PTSD total and symptom cluster severity scores; participant characteristics such as average age, sex, race and education; study methodology including sample size and study design; potential predictor factors (eg, APOE genotype, prisoner of war status, pharmaceutical medications, cognitive training interventions and health behaviours); study findings (eg, ORs and HRs) and author conclusions. Disagreements between the two reviewers will be resolved by consensus or by obtaining a third reviewer's opinion should the two reviewers fail to reach consensus.

\section{Assessment of study bias}

Recommendations from Grading of Recommendations, Assessment, Development and Evaluations will be followed to allow assessment of risk-of-bias, imprecision, inconsistency, indirectness, publication bias, magnitude of effect, dose-response gradient and residual confounding. ${ }^{43}$ This approach gives a preliminary score of low to observational studies and high to RCTs. ${ }^{37}$ This preliminary score can be upgraded by a thorough study design, constancy, accuracy and other criteria as described by Williams and colleagues. $^{37}$

\section{Analysis}

If studies are of sufficient homogeneity regarding participants, interventions and outcomes, a meta-analysis will be conducted. ${ }^{44}$ In accordance with the guidelines for quantitative synthesis of systematic review evidence, ${ }^{45}$ a tabular summary of findings will be created. This table will include extracted variables, information regarding the quality of 
evidence, and the scale of effect of any interventions and outcomes investigated. For this study, the outcomes are associations of risk and protective factors with MCI or dementia in adults with PTSD.

The purpose of the quantitative synthesis is to integrate quantitative findings across studies to achieve a coherent synthesis. ${ }^{45}$ A quantitative analysis can be performed through a meta-analysis by computing a quantitative summary measure. ${ }^{46}$ For each study, a single effect size will be computed for each risk/protective factor and its association with MCI/dementia. Pearson's $r$ will be used for computation of global effect sizes. ORs or risk ratios will be converted into Pearson's $r$ to estimate the global effect. In this way, the $r$ statistic can be used to describe the association between specific risk and protective factors and MCI/dementia. ${ }^{47}$ Cohen's guidelines will be used to interpret the magnitude of the effect: $0.10=$ small, $0.30=$ medium and $0.50=$ large ${ }^{48} \mathrm{~A}$ random-effects model will be used for the estimation of the global effect. The Q-statistic will be used to calculate heterogeneity of effect sizes across studies. In addition, the $\mathrm{I}^{2}$ statistic will allow computation of the proportion of the observed heterogeneity that is due to real variation in effect sizes across studies versus that due to sampling error.

Based on a previous systematic review of risk and protective factors for dementia, ${ }^{10}$ included studies may differ regarding participants and/or outcomes resulting in clinical and statistical heterogeneity. In that case, a meta-analytic approach is not recommended in order to avoid reporting ambiguous values, ${ }^{44}$ and a narrative approach will be conducted. The narrative approach will follow the guidelines of the Cochrane Handbook ${ }^{49}$ and will also incorporate the graphical presentation of data in the form of a forest plot, to allow visual analysis of the patterns of included studies. ${ }^{5051}$

\section{Ethics and dissemination}

The proposed study will not collect individual-level data and, therefore, does not require ethical approval. The results of this systematic review will provide the most up to date literature synthesis of risk and protective factors of dementia in adults with PTSD. Results will be disseminated through peer-reviewed publications and presentations at relevant national and international conferences.

\section{DISCUSSION}

In the USA, one-third of seniors die with dementia. Annually, dementia-related care costs have reached US $\$ 290$ billion. ${ }^{52}$ Epidemiological research has revealed that PTSD is associated with increased risk for dementia. Even after controlling for comorbidities (eg, head injury) and other risk factors (eg, smoking), military veterans, ${ }^{56}$ first-responders, ${ }^{17}$ and civilians ${ }^{89}$ with PTSD showed an approximately twofold ${ }^{5}$ and fourfold ${ }^{9}$ greater risk of developing all-type dementia, respectively.

While it has been well established that PTSD is associated with impaired cognitive performance, ${ }^{12-16}$ whether the neurobiological changes associated with PTSD account for increased dementia risk is a significant gap in knowledge. This systematic review will provide a comprehensive search of the literature regarding risk and protective factors of dementia among adults with PTSD.

In the case of $\mathrm{AD}$, early alterations in the brain can be detected by imaging and other biomarkers, ${ }^{18-20}$ creating the potential opportunity for being able to target modifiable risk and protective factors of Alzheimer's dementia. Implementation of screening and detection of cognitive decline to facilitate early detection and delay or prevention of $\mathrm{MCI} /$ non-specific dementia in the PTSD population is another area in need of research.

Contributors All authors made substantial contributions to this paper. KAL supervised all aspects of the study design and took responsibility for the paper as a whole. BLP oversaw the study design and search strategy. KAL, TP and SH developed the search strategy. KAL drafted the manuscript. KAL, TMP, MML, DLS and BLP contributed substantially to the revision of the final manuscript.

Funding The work was supported by the Building Interdisciplinary Research Careers in Women's Health (BIRCWH) grant (\#5K12DA035150) from the Office of Research on Women's Health (ORWH) and National Institute on Drug Abuse (NIDA) at the National Institutes of Health (NIH).

\section{Competing interests None declared.}

Patient and public involvement Patients and/or the public were not involved in the design, or conduct, or reporting, or dissemination plans of this research.

Patient consent for publication Not required.

Provenance and peer review Not commissioned; externally peer reviewed.

Open access This is an open access article distributed in accordance with the Creative Commons Attribution Non Commercial (CC BY-NC 4.0) license, which permits others to distribute, remix, adapt, build upon this work non-commercially, and license their derivative works on different terms, provided the original work is properly cited, appropriate credit is given, any changes made indicated, and the use is non-commercial. See: http://creativecommons.org/licenses/by-nc/4.0/.

\section{ORCID iDs}

Karen A Lawrence http://orcid.org/0000-0002-7235-5594

Molly M Long http://orcid.org/0000-0001-5302-6659

\section{REFERENCES}

1 Greenberg MS, Tanev K, Marin M-F, et al. Stress, PTSD, and dementia. Alzheimers Dement 2014;10:S155-65.

2 Association AP. Diagnostic and statistical manual of mental disorders. 5 edn. Arlington, VA, 2013.

3 Kessler RC, Petukhova M, Sampson NA, et al. Twelve-month and lifetime prevalence and lifetime morbid risk of anxiety and mood disorders in the United States. Int J Methods Psychiatr Res 2012;21:169-84.

4 Bureau USC. Annual estimates of resident population by sex and selected age groups for the United States, 2012 ..

5 Kang B, Xu H, McConnell ES. Neurocognitive and psychiatric comorbidities of posttraumatic stress disorder among older veterans: a systematic review. Int J Geriatr Psychiatry 2019;34:511-2.

6 Yaffe K, Vittinghoff E, Lindquist K, et al. Posttraumatic stress disorder and risk of dementia among US veterans. Arch Gen Psychiatry 2010;67:608-13.

7 Qureshi SU, Kimbrell T, Pyne JM, et al. Greater prevalence and incidence of dementia in older veterans with posttraumatic stress disorder. J Am Geriatr Soc 2010;58:1627-33.

8 Flatt JD, Gilsanz P, Quesenberry CP, et al. Post-traumatic stress disorder and risk of dementia among members of a health care delivery system. Alzheimers Dement 2018;14:28-34.

9 Wang T-Y, Wei H-T, Liou Y-J, et al. Risk for developing dementia among patients with posttraumatic stress disorder: a nationwide longitudinal study. J Affect Disord 2016;205:306-10. 
10 Plassman BL, Williams JW, Burke JR, et al. Systematic review: factors associated with risk for and possible prevention of cognitive decline in later life. Ann Intern Med 2010;153:182-93.

11 Daviglus ML, Plassman BL, Pirzada A, et al. Risk factors and preventive interventions for Alzheimer disease: state of the science. Arch Neurol 2011;68:1185-90.

12 Yehuda R, Golier JA, Halligan SL, et al. Learning and memory in holocaust survivors with posttraumatic stress disorder. Biol Psychiatry 2004;55:291-5.

13 Yehuda R, Golier JA, Tischler L, et al. Learning and memory in aging combat veterans with PTSD. J Clin Exp Neuropsychol 2005;27:504-15

14 Yehuda R, Keefe RS, Harvey PD, et al. Learning and memory in combat veterans with posttraumatic stress disorder. Am J Psychiatry 1995;152:137-9.

15 Yehuda R, Tischler L, Golier JA, et al. Longitudinal assessment of cognitive performance in holocaust survivors with and without PTSD. Biol Psychiatry 2006;60:714-21.

16 Schuitevoerder S, Rosen JW, Twamley EW, et al. A meta-analysis of cognitive functioning in older adults with PTSD. J Anxiety Disord 2013;27:550-8.

17 Clouston SAP, Kotov R, Pietrzak RH, et al. Cognitive impairment among world Trade center responders: long-term implications of re-experiencing the 9/11 terrorist attacks. Alzheimers Dement 2016;4:67-75.

18 Jack CR, Knopman DS, Jagust WJ, et al. Tracking pathophysiological processes in Alzheimer's disease: an updated hypothetical model of dynamic biomarkers. Lancet Neurol 2013:12:207-16

19 Jack CR, Therneau TM, Wiste HJ, et al. Transition rates between amyloid and neurodegeneration biomarker states and to dementia: a population-based, longitudinal cohort study. Lancet Neurol 2016;15::56-64.

20 Jack CR, Wiste HJ, Weigand SD, et al. Defining imaging biomarker cut points for brain aging and Alzheimer's disease. Alzheimers Dement 2017;13:205-16.

21 Cook JM, Simiola V. Trauma and PTSD in older adults: prevalence, course, concomitants and clinical considerations. Curr Opin Psychol 2017:14:1-4.

22 McKhann GM, Knopman DS, Chertkow H, et al. The diagnosis of dementia due to Alzheimer's disease: recommendations from the national institute on Aging-Alzheimer's association workgroups on diagnostic guidelines for Alzheimer's disease. Alzheimers Dement 2011;7:263-9.

23 Rytwinski NK, Scur MD, Feeny NC, et al. The co-occurrence of major depressive disorder among individuals with posttraumatic stress disorder: a meta-analysis. J Trauma Stress 2013;26:299-309.

24 Blanco C, Xu Y, Brady K, et al. Comorbidity of posttraumatic stress disorder with alcohol dependence among US adults: results from national epidemiological survey on alcohol and related conditions. Drug Alcohol Depend 2013;132:630-8.

25 Farr OM, Sloan DM, Keane TM, et al. Stress- and PTSDassociated obesity and metabolic dysfunction: a growing problem requiring further research and novel treatments. Metabolism 2014;63:1463-8

26 Mota NP, Han S, Harpaz-Rotem I, et al. Apolipoprotein E gene polymorphism, trauma burden, and posttraumatic stress symptoms in U.S. military veterans: results from the National health and resilience in veterans study. Depress Anxiety 2018;35:168-77.

27 Olff M. Sex and gender differences in post-traumatic stress disorder: an update. Eur J Psychotraumatol 2017;8:1351204.

28 Au B, Dale-McGrath S, Tierney MC. Sex differences in the prevalence and incidence of mild cognitive impairment: a metaanalysis. Ageing Res Rev 2017;35:176-99.
29 Cosgrove KP, Mazure CM, Staley JK. Evolving knowledge of sex differences in brain structure, function, and chemistry. Biol Psychiatry 2007;62:847-55.

30 Bowman K, Thambisetty M, Kuchel GA, et al. Obesity and longer term risks of dementia in 65-74 year olds. Age Ageing 2019;48:367-73.

31 Byers AL, Yaffe K. Depression and risk of developing dementia. Nat Rev Neurol 2011;7:323-31.

32 Bertram L, McQueen MB, Mullin K, et al. Systematic meta-analyses of Alzheimer disease genetic association studies: the AlzGene database. Nat Genet 2007;39:17-23.

33 Farrer LA, Cupples LA, Haines JL, et al. Effects of age, sex, and ethnicity on the association between apolipoprotein $E$ genotype and Alzheimer disease. A meta-analysis. APOE and Alzheimer disease meta analysis consortium. JAMA 1997;278:1349-56.

34 Guo Z, Fratiglioni L, Viitanen M, et al. Apolipoprotein E genotypes and the incidence of Alzheimer's disease among persons aged 75 years and older: variation by use of antihypertensive medication? Am J Epidemiol 2001;153:225-31.

35 Slooter AJ, Cruts M, Kalmijn S, et al. Risk estimates of dementia by apolipoprotein $E$ genotypes from a population-based incidence study: the Rotterdam study. Arch Neurol 1998;55:964-8.

36 Takeda M, Martínez R, Kudo T, et al. Apolipoprotein E and central nervous system disorders: reviews of clinical findings. Psychiatry Clin Neurosci 2010;64:592-607.

37 Williams JW, Plassman BL, Burke J, et al. Preventing Alzheimer's disease and cognitive decline. Evid Rep Technol Assess

38 Moher D, Shamseer L, Clarke M, et al. Preferred reporting items for systematic review and meta-analysis protocols (PRISMA-P) 2015 statement. Syst Rev 2015;4:1.

39 Launer LJ. The epidemiologic study of dementia: a life-long quest? Neurobiol Aging 2005;26:335-40.

40 Salthouse TA. When does age-related cognitive decline begin? Neurobiol Aging 2009;30:507-14.

41 Association AP. Diagnostic and statistical manual of mental disorders : DSM-III. 3 edn. Washington DC: American Psychiatric Association, 1980.

42 Veritas Health Innovation. Covidence systematic review software [program]. Melbourne, Australia: Veritas Health Innovation, 2014.

43 Schünemann HJ, Cuello C, Akl EA, et al. Grade guidelines: 18. How ROBINS-I and other tools to assess risk of bias in nonrandomized studies should be used to rate the certainty of a body of evidence. $J$ Clin Epidemiol 2019;111:105-14.

44 Deeks J, Higgins J, Altman D. Analysing data and undertaking meta-analyses. In: Cochrane handbook for systematic reviews of interventions [Internet]. 2019. London: Cochrane, 2019.

45 Higgins JPT, López-López JA, Becker BJ, et al. Synthesising quantitative evidence in systematic reviews of complex health interventions. BMJ Glob Health 2019;4:e000858.

46 Petticrew M, Roberts H. Systematic reviews in the social sciences: a practical guide. Malden, MA: Blackwell, 2006.

47 Bonett DG. Transforming odds ratios into correlations for metaanalytic research. Am Psychol 2007:62:254-5.

48 Cohen J. A power primer. Psychol Bull 1992;112:155-9.

49 Higgins JPT, Green S, Cochrane Collaboration. Cochrane handbook for systematic reviews of interventions. Chichester, England ; Hoboken, NJ: Wiley-Blackwell, 2008.

50 McKenzie J, Brennan S, Ryan R. Summarizing study characteristics and preparing for synthesis. In: Cochrane handbook for systematic reviews of interventions version 60, 2019.

51 Popay J, Roberts H, Sowden A, et al. Guidance on the conduct of narrative synthesis in systematic reviews: a product of the ESRC methods programme. Lancaster, UK: University of Lancaster, 2006.

52 Alzheimer's Association. 2014 Alzheimer's disease facts and figures. Alzheimers Dement 2014;10:e47-92. 